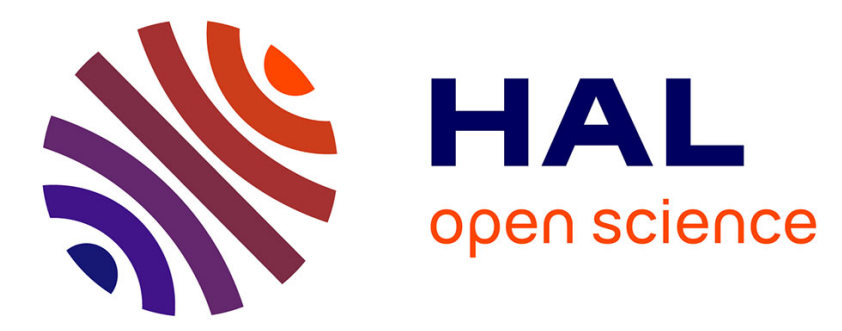

\title{
Multirate coupling of controlled rectifier and non-linear finite element model based on Waveform Relaxation Method
}

Antoine Pierquin, Thomas Henneron, Stephane Brisset, Stéphane Clenet

\section{To cite this version:}

Antoine Pierquin, Thomas Henneron, Stephane Brisset, Stéphane Clenet. Multirate coupling of controlled rectifier and non-linear finite element model based on Waveform Relaxation Method. IEEE Transactions on Magnetics, 2016, 52 (3), pp.1-4. 10.1109/TMAG.2015.2489762 . hal-01273228

\author{
HAL Id: hal-01273228 \\ https://hal.science/hal-01273228
}

Submitted on 12 Feb 2016

HAL is a multi-disciplinary open access archive for the deposit and dissemination of scientific research documents, whether they are published or not. The documents may come from teaching and research institutions in France or abroad, or from public or private research centers.
L'archive ouverte pluridisciplinaire HAL, est destinée au dépôt et à la diffusion de documents scientifiques de niveau recherche, publiés ou non, émanant des établissements d'enseignement et de recherche français ou étrangers, des laboratoires publics ou privés. 


\title{
Multirate coupling of controlled rectifier and non-linear finite element model based on Waveform Relaxation Method
}

\author{
A. Pierquin ${ }^{1}$, T. Henneron ${ }^{1}$, S. Brisset ${ }^{2}$ and S. Clénet $^{3}$ \\ ${ }^{1}$ L2EP, Université de Lille 1, Cité Scientifique, 59655 Villeneuve d'Ascq, France \\ ${ }^{2}$ L2EP, EC Lille, Cité Scientifique, CS 20048, 59651 Villeneuve d'Ascq, France \\ ${ }^{3}$ L2EP, Arts et Métiers ParisTech, 59046 Lille Cedex, France
}

\begin{abstract}
To study a multirate system, each subsystem can be solved by a dedicated sofware with respect to the physical problem and the time constant. Then, the problem is the coupling of the solutions of the subsystems. The Waveform Relaxation Method (WRM) seems to be an interesting solution for the coupling but until now it has been mainly applied on academic examples. In this paper, the WRM is applied to perform the coupling of a controlled rectifier and a non-linear finite element model of a transformer.
\end{abstract}

Index Terms-Waveform relaxation method, multirate system, multi-physics and coupled problems, finite element method.

\section{INTRODUCTION}

A $\mathrm{N}$ electrical system can involve several devices with different physics and dynamics. The simulation in the time domain of such a set of devices can be carried out by coupling numerical models that is to say a system of Differential Algebraic Equations (DAE).

A direct strong coupling consists in gathering the DAE systems and to solve them at the same time. With an indirect strong coupling, each DAE system is solved successively until convergence at each time step. But in both cases, the timestep must be chosen according to the smallest time constant. This can lead to an expensive computation time if some DAE systems have a large number of unknowns like in the case of Finite Element Model (FEM). A first alternative can be the weak coupling of the DAE systems with different time-steps, but the consistency of the coupling is most of the time not assured. A second alternative is to reduce the FEM using a surface response technique [1]. The issue is then to control the accuracy of the reduced model.

The Waveform Relaxation Method (WRM) [2] allows a consistent coupling to solve this kind of problem. The WRM approach is an iterative process which converges in theory to the solution of a strong coupling [3]. The method was used for many years for the simulation of circuits [4], [5] or transmission lines [6], and has been recently studied for the coupling of circuit equations and a FEM [7], [8], either by source coupling or by parameter coupling [9], [10].

In this paper, we propose to study the parameter coupling of a FEM to circuit equations including a control loop. First, the WRM is briefly described. Then, the interest and the basic principle of the method is brought out on academic example. Afterwards, a more elaborate WRM approach is applied to study a more realistic and complex system involving a control loop, a single phase transformer associated with a controlled rectifier. The transformer is modeled by a non-linear FEM and the rectifier is controlled by a Pulse Width Modulation (PWM) technique, each subsystem being studied with a time-step adapted to its time constant. We use the parameter coupling
WRM to allow not only the coupling between the rectifier and the FEM, but also the control of the current into the FEM.

\section{WAVEFORM RELAXATION METHOD}

Let consider a system composed of $r$ subsystems, each subsystem $i$ satisfying the DAE

$$
\begin{aligned}
\dot{\mathbf{y}}_{i}(t) & =\mathbf{f}_{i}(\mathbf{y}(t), \mathbf{z}(t)) \\
0 & =\mathbf{g}_{i}(\mathbf{y}(t), \mathbf{z}(t)),
\end{aligned}
$$

with $t \in[0, T]$ and the initial conditions $\mathbf{y}(0)=\mathbf{y}_{0}$ and $\mathbf{z}(0)=$ $\mathbf{z}_{0}, \mathbf{y}$ being the differential variables and $\mathbf{z}$ the algebraic variables. The WRM computes iteratively an approximation $\left(\tilde{\mathbf{y}}^{k}(t), \tilde{\mathbf{z}}^{k}(t)\right)$ of the exact solution. The first step is the extrapolation step: for $k=0, \tilde{\mathbf{y}}^{k}(t)=\mathbf{y}_{0}, \tilde{\mathbf{z}}^{k}(t)=\mathbf{z}_{0}, \forall t \in[0, T]$. Then at the iteration $k$ and for the subsystem $i$, the algorithm solves

$$
\begin{aligned}
\dot{\tilde{\mathbf{y}}}_{i}^{k}(t) & =\mathbf{f}_{i}\left(\mathbf{Y}_{i}^{k}(t), \mathbf{Z}_{i}^{k}(t)\right) \\
0 & =\mathbf{g}_{i}\left(\mathbf{Y}_{i}^{k}(t), \mathbf{Z}_{i}^{k}(t)\right) .
\end{aligned}
$$

The value of $\mathbf{Y}_{i}^{k}$ (resp. $\mathbf{Z}_{i}^{k}$ ) depends on $\tilde{\mathbf{y}}^{k-1}$ and $\tilde{\mathbf{y}}^{k}$ (resp. $\tilde{\mathbf{z}}^{k-1}$ and $\tilde{\mathbf{z}}^{k}$ ) and on the relaxation schemes (Picard, Jacobi or Gauss-Seidel). For example, with the GaussSeidel scheme, the subsystems are solved sequentially with $\mathbf{Y}_{i}^{k}(t)=\left[\tilde{\mathbf{y}}_{1}^{k}, \ldots, \tilde{\mathbf{y}}_{i-1}^{k}, \tilde{\mathbf{y}}_{i}^{k}, \tilde{\mathbf{y}}_{i+1}^{k-1}, \ldots, \tilde{\mathbf{y}}_{r}^{k-1}\right]^{\mathrm{T}}$, and $\mathbf{Z}_{i}^{k}(t)=$ $\left[\tilde{\mathbf{z}}_{1}^{k}, \ldots, \tilde{\mathbf{z}}_{i-1}^{k}, \tilde{\mathbf{z}}_{i}^{k}, \tilde{\mathbf{z}}_{i+1}^{k-1}, \ldots, \tilde{\mathbf{z}}_{r}^{k-1}\right]^{\mathrm{T}}$.

Subsystems are solved on the overall time domain $[0, T]$, then the waveforms are transfered from one subsystem to the others. Consequently, each subsystem can be solved with respect to its own time-step. Since the waveforms $\mathbf{y}_{i}$ and $\mathbf{z}_{i}$ of each subsystem are sampled with different time-steps, interpolation technics are required to reconstruct a waveform consistent with the other time steps.

\section{ACADEMIC PROBLEM}

To introduce the problematic of the targeted applications and the interests of the WRM, we consider the coupling of a LC filter with a transformer. The LC filter contains a PWM voltage 
source and supplies the transformer with a filtered voltage. To simulate such a device, a very small time-step is necessary to deal with the PWM. As the amplitude of high order harmonics of the voltage applied to the transformer are very small due to the LC filter, the time-step could be larger for this part of the device. If a finite element model of the transformer is considered with the smallest time-step, the computation time can be extremely high. A strong coupling is so inappropriate in this case. On the contrary, the waveform relaxation approach seems well-adapted to simulate the device since a time-step adapted to each subsystem can be used.

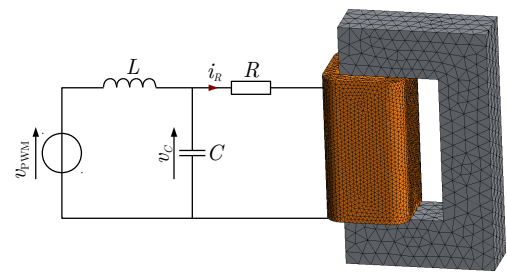

(a) Complete device: strong coupling

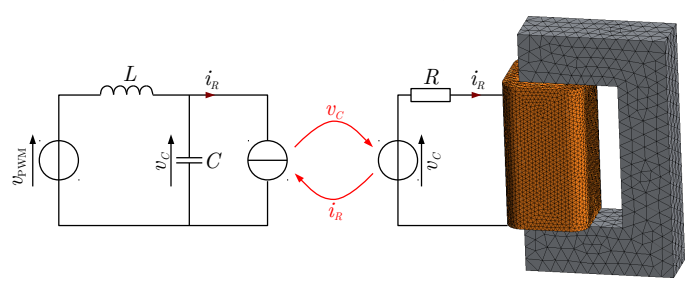

(b) Spit device: WRM source coupling

Fig. 1. Strong coupling and WRM models of the device.

The PWM voltage source has a $50 \mathrm{~Hz}$ fondamental frequency and a $20 \mathrm{kHz}$ switching frequency. As a first step, the model in Fig. 1(a) is solved with a strong coupling by a FEM. The resulting voltage and currents are presented in Fig. 2 to 4. The current $i_{R}$ appears to be a smooth and regular waveform with a $50 \mathrm{~Hz}$ frequency.

As a second step, the device is split in two subsystems with the LC filter on one hand, the FEM on the other hand (Fig. 1(b)). The LC circuit is simulated with a time-step adapted to the PWM $\left(2.10^{-7} \mathrm{~s}\right)$, and the FEM with a time-step 500 times larger. The coupling is done by supplying the primary coil of the transformer by the interpolated voltage $v_{c}$ from the circuit; then the current $i_{R}$ in the winding is interpolated and imposed to the circuit as a source. This kind of WRM is called source coupling. The interpolation is a simple linear interpolation between two points.

The results plotted on the same Fig. 2 to 4 show quite good similarity. The introduction of a coarse time-stepping for the transformer and of the interpolation leads to a waste of precision. Nevertheless, the error indicated in Table I are really weak. Then, after $6 \mathrm{WRM}$ iterations, the error is less than $1.23 \%$. The convergence of the process is very fast, as it can be seen on Fig. 5: after 6 iterations, the criterion convergence is less than $10^{-7}$. The most important information to notice is that the computation time to simulate only one period is very long with the strong coupling. Several weeks are needed to obtain results, whereas with the WRM the results are available in several hours (Table I).

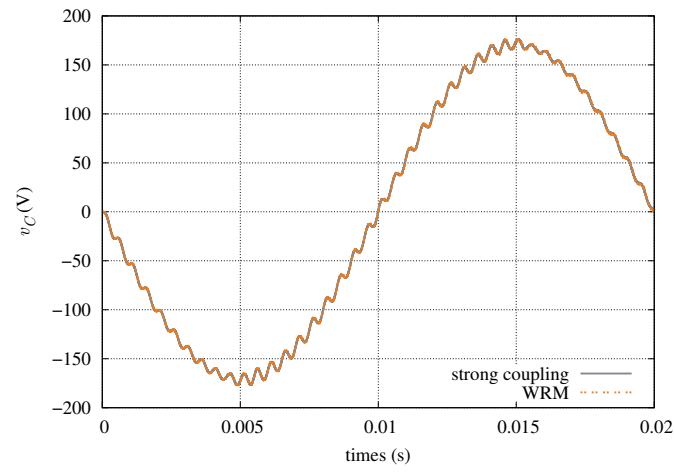

Fig. 2. Voltage $v_{c}$.

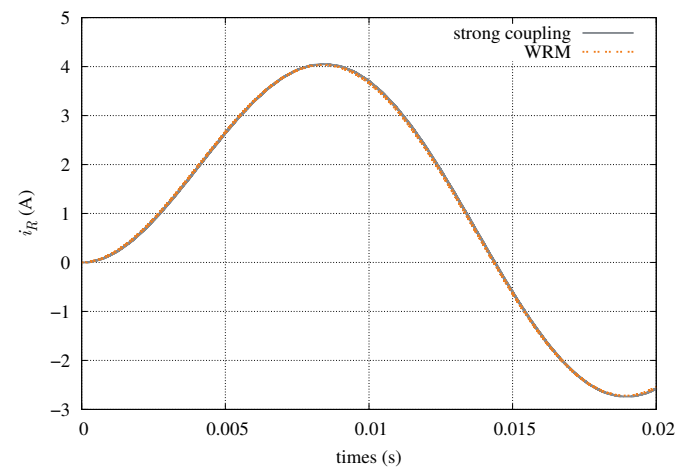

Fig. 3. Current $i_{R}$.

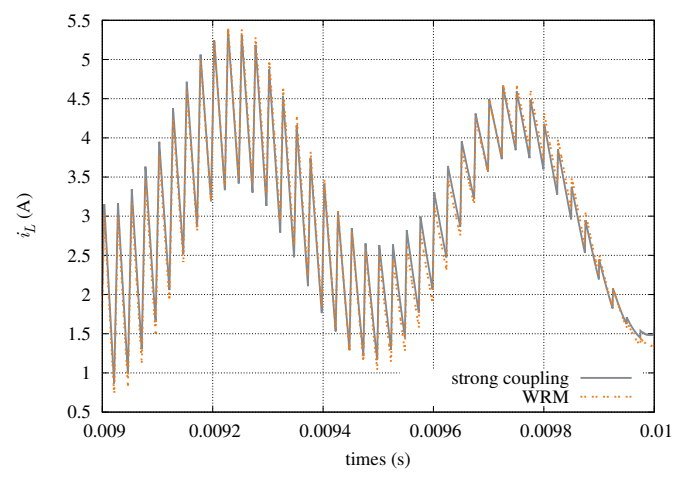

Fig. 4. Current $i_{L}$.

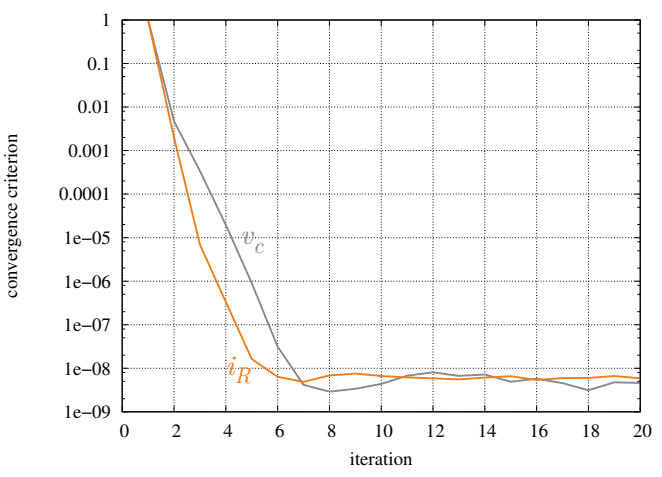

Fig. 5. Convergence criterion of the WRM. 
The computation time is considerably reduced with the WRM because the number of arithmetic operations itself is reduced compared to a strong coupling. We call $n_{u 1}$ (respectively $n_{u 2}$ ) the number of unknowns of the FE problem (resp. the circuit problem) and $n_{t 1}$ (respectively $n_{t 2}$ ) the number of time-steps of the FE problem (resp. the circuit problem). The conjugate gradient (CG) is used to solve the matrix system of the FE problem. The complexity of the $\mathrm{CG}$, including convergence, is in $\mathcal{O}\left(n_{u 1}^{3}\right)$ for a full matrix $\left(\mathcal{O}\left(n_{u 1}^{2}\right)\right.$ per iteration with a convergence in $n_{u 1}$ iterations at the most) or $\mathcal{O}\left(n_{u 1}^{\alpha}\right), \alpha<2$ for a sparse matrix. In our problem, $n_{u 1} \gg n_{u 2}$ and $n_{t 2}>n_{t 1}$, so we can consider a complexity of $\mathcal{O}\left(n_{t 2} n_{u 1}^{\alpha}\right)$ for a strong coupling and $\mathcal{O}\left(K n_{t 1} n_{u 1}^{\alpha}\right)$ for a WRM coupling, with $K$ the maximum number of WRM iterations. As $n_{t 2}>>n_{t 1}$, in most of the case, $n_{t 2}>K n_{t 1}$ and so the computation time could be reduced.

TABLE I

COMPUTATION TIME, COMPLEXITY AND ERRORS FOR 6 WRM ITERATIONS

\begin{tabular}{lcc}
\hline \hline & Strong coupling & WRM coupling \\
\hline Computation time & 13 days 7h8min & $2 \mathrm{~h} 40 \mathrm{~min}$ \\
Error on $i_{R}$ & - & $1.13 \%$ \\
Error on $v_{c}$ & - & $0.67 \%$ \\
\hline \hline
\end{tabular}

\section{INDUSTRIAL APPLICATION}

The WRM is applied to study a more complex device. Let us consider a transformer and its associated rectifier as in Fig. 6. The rectifier is controlled to provide a direct voltage $v_{\mathrm{dc}}$ of 800 $\mathrm{V}$ and a current $i_{s}$ into the secondary winding in phase with the voltage $v_{20}$; moreover, a current $i_{\mathrm{ch}}$ is imposed into the rectifier circuit as a load current. The control based on a PWM requires a very small time-step $\Delta t_{r}=5 \cdot 10^{-6}$ s. The transformer is modeled by a FE method with a magnetic vector potential formulation. This model is non-linear: the reluctivity depends on the magnetic field. The voltages $v_{10}$ and $v_{s}$ are respectively imposed to the primary and secondary windings. The solution of the FEM using the time-step $\Delta t_{r}$ is unaffordable in terms of computation time. The WRM allows to use a larger time-step allowing to drastically reduce the computation time.

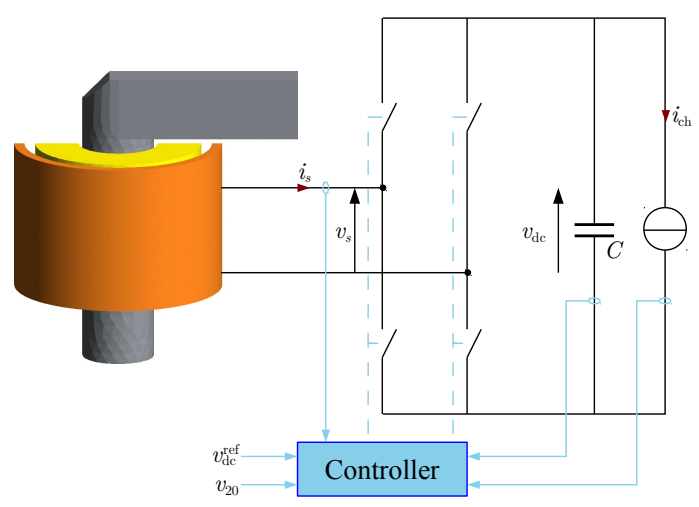

Fig. 6. Complete device of the traction transformer
The WRM is applied to perform the coupling between the FEM of the transformer and the circuit model of the rectifier. A classic source coupling, as the one used in the previous section, does not allow to control the current $i_{s}$ into the secondary winding because it would be imposed as a source to the circuit rectifier part. Therefore, a parameter coupling is used [9], [10]. An equivalent circuit of the transformer is introduced into the rectifier circuit (Fig.7) with a resistance $R$ and an inductance $L$. The resistance is the same than in the FEM, and the inductance is obtained by a calculation based on a linear FEM of the transformer. Then, a residual current source $i_{\text {res }}$ is added (Fig. 7) to guarantee the consistency of the coupling. The non-linear effects of the tranformer are introduced into the electrical circuit by the residual current, without any change in the equivalent model.

At the $k^{\text {th }}$ iteration of the WRM process, the source of the FEM is the voltage $v_{s}^{k}$, and its solution provides the current $i_{s}^{k}$ in the secondary winding. The voltage $v_{s}^{k}$ is given by the solution of the electrical circuit model composed of the equivalent circuit of the transformer and the controlled rectifier. In this model, the residual current $i_{\text {res }}^{k}$ is a source; the voltage $v_{\mathrm{dc}}^{k}$ is controlled to be equal to $800 \mathrm{~V}$ and the current $i_{2}^{k}$ is also controlled to be in phase with the voltage $v_{20}$. The residual current is such that $i_{\text {res }}^{k}=i_{s}^{k-1}-i_{L}^{k-1}$. Over the iterations of the WRM process, the current $i_{s}^{k}$ converges to $i_{2}^{k}$. At the end of the process, $i_{s}^{k}$ is in phase with the voltage $v_{20}$. Fig. 8 presents the convergence criterion related to the current $i_{s}$ of the WRM process. The convergence is less clear compared to the academic example. Nevertheless, the WRM gives solutions relatively stable. According to the parameter coupling, the current $i_{s}^{k}$ tends to be in phase with the voltage $v_{20}$ (Fig. 9). Furthermore, Fig. 11 shows that the voltage $v_{\mathrm{dc}}$ is close to $800 \mathrm{~V}$. Fig. 10 shows the current $i_{p}$ into the primary winding for non-linear FEM.

To compute this simulation on a time interval of 2 seconds, 3 days 9 hours 31 minutes are needed to perform 4 WRM iterations, but $99.81 \%$ of the computation time is dedicated to solve the FEM. This should be compared with the 3 months estimated to perform the simulation using the same time step for the control loop and the FEM. The WRM converges to the exact solution as proved in [9], so the solution is supposed to be close to the exact one.

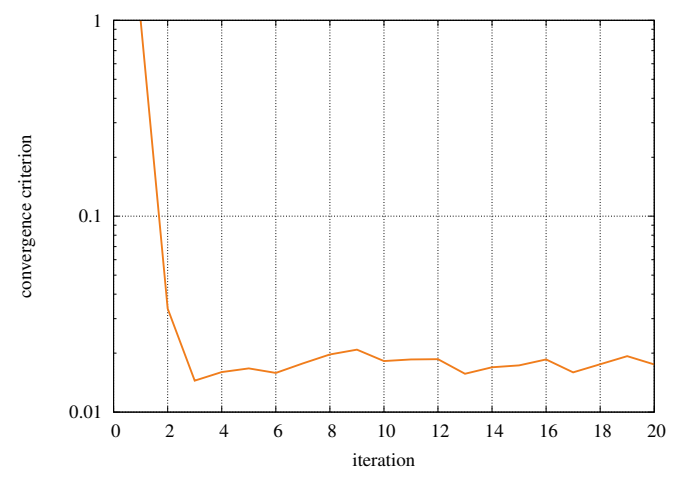

Fig. 8. Convergence criterion of the WRM for the traction transformer. 


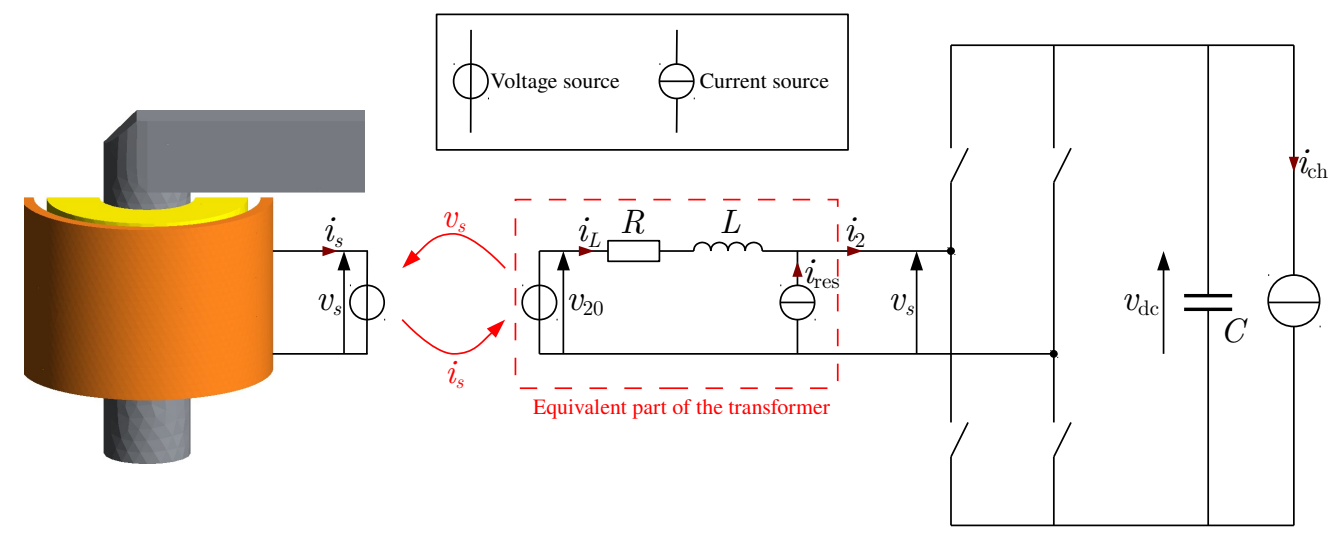

Fig. 7. WRM parameter coupling for the traction transformer.

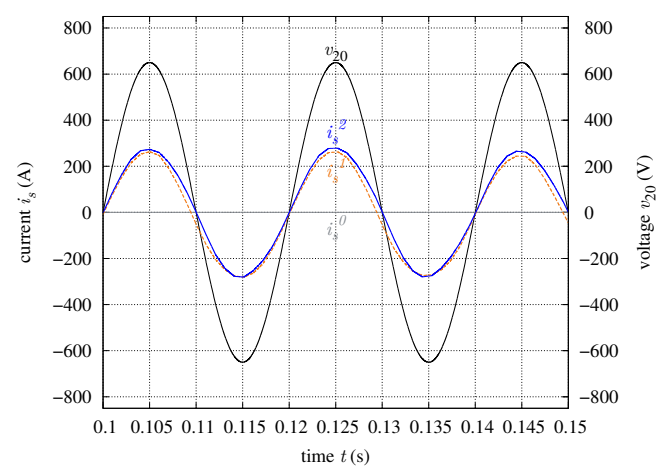

Fig. 9. Current $i_{s}^{k}$ with respect to the WRM iterations and voltage $v_{20}$.

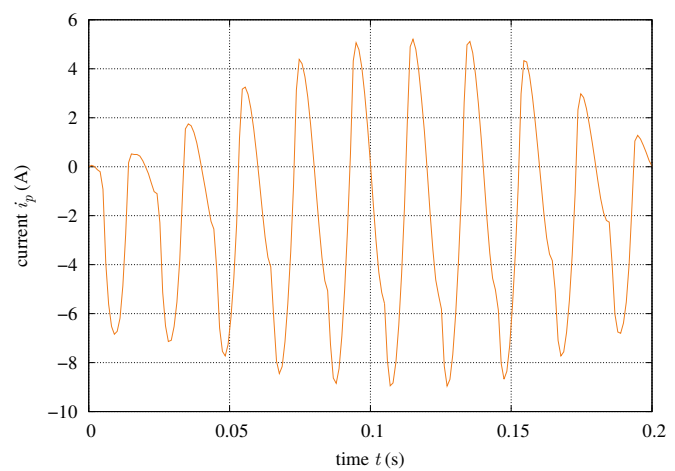

Fig. 10. Current $i_{p}$ into the primary winding for the non-linear FEM.

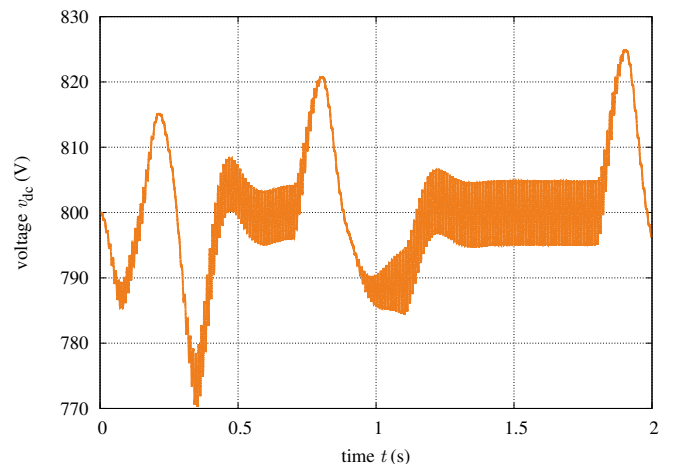

Fig. 11. DC-bus voltage $v_{\mathrm{dc}}$.

\section{CONCLUSION}

The WRM is well-adapted to the coupling and the simulation of multirate systems because it allows to use a time discretisation per subsystem.. A first application presented the source coupling approach on an academic case. Comparisons with a strong coupling showed small errors and a speed up of 120. A second application showed the interest of the parameter coupling for control the current in a non-linear FEM coupled to a rectifier.

\section{REFERENCES}

[1] J. R. Brauer, Magnetic Actuators and Sensors, 2nd Edition, march 2014, ch. 15.1 .3 , pp. 292-293.

[2] E. Lelarasmee, A. Ruehli, and A. Sangiovanni-Vincentelli, "The waveform relaxation method for time-domain analysis of large scale integrated circuits," Computer-Aided Design of Integrated Circuits and Systems, IEEE Transactions on, vol. 1, no. 3, pp. 131 - 145, july 1982.

[3] M. Arnold and M. Gunther, "Preconditioned dynamic iteration for coupled differential-algebraic systems," Bit Numerical Mathematics, vol. 41 , no. 1 , pp. $1-25,2001$.

[4] M. Crow, M. Ilic, and J. White, "Convergence properties of the waveform relaxation method as applied to electric power systems," Circuits and Systems, 1989., IEEE International Symposium on, vol. 3, pp. 1863 -1866 , may 1989.

[5] F. Cingoz, H. Behjati, and A. Davoudi, "Accelerated simulation of ultracapacitors using waveform relaxation method," in Transportation Electrification Conference and Expo (ITEC), 2012 IEEE, June 2012, pp. 1-6.

[6] F.-Y. Chang, "Waveform relaxation analysis of rlcg transmission lines," Circuits and Systems, IEEE Transactions on, vol. 37, no. 11, pp. 13941415, Nov 1990.

[7] S. Schöps, H. De Gersem, and A. Bartel, "A cosimulation framework for multirate time integration of field/circuit coupled problems," Magnetics, IEEE Transactions on, vol. 46, no. 8, pp. 3233 -3236, aug. 2010.

[8] J. d. D. Nshimiyimana, F. Plumier, P. Dular, and C. Geuzaine, "Optimized waveform relaxation methods for modeling electromagnetic fieldcircuit problems," in Proceedings of CEFC, may 2014.

[9] A. Bartel, M. Brunk, M. Günther, and S. Schöps, "Dynamic iteration for coupled problems of electric circuits and distributed devices," SIAM Journal on Scientific Computing, vol. 35, no. 2, pp. B315-B335, 2013.

[10] G. Alì, A. Bartel, M. Brunk, and S. Schöps, "A convergent iteration scheme for semiconductor/circuit coupled problems," in Scientific Computing in Electrical Engineering SCEE 2010, ser. Mathematics in Industry, B. Michielsen and J.-R. Poirier, Eds. Springer Berlin Heidelberg, 2012, pp. 233-242. 